\title{
15 Fragenkatalog des TMF-Rechtsgutachtens aus dem Pflichtenheft
}

Die ausführliche Begründung der Antworten zu den Fragen im Fragenkatalog finden sich in den mit einem „\#”“ angezeigten Kapiteln des obigen Gutachtens.

\section{Rechtsgrundlagen}

1. Verhältnis der Rechtsgrundlagen aus Art. 6 und Art. 9 EU-Datenschutzgrundverordnung (im Folgenden: DSGVO):

1.1 Inwiefern stellen die Rechtsgrundlagen in Art. 9 DSGVO eigenständige Rechtsgrundlagen dar oder sind sie immer nur als zusätzliche, einschränkende Anforderungen in Bezug auf eine notwendige (aber eben im Falle der von Art. 9 DSGVO erfassten Daten nicht hinreichende) Rechtsgrundlage nach Art. 6 DSGVO anzusehen?

Bei der Verarbeitung besonderer Kategorien personenbezogener Daten muss ergänzend zu den Erlaubnistatbeständen des Art. 9 Abs. 2 DSGVO ein Erlaubnistatbestand des Art. 6 Abs. 1 UAbs. 1 DSGVO vorliegen. Im Anwendungsbereich der Öffnungsklauseln in Art. 9 Abs. 2 lit. b, g, h, i, j DSGVO erlauben aufgrund dieser Vorschriften erlassene Spezialvorschriften der Union oder der Mitgliedstaaten die Datenverarbeitung. (III Kap. 4.2-4.5)

1.2 Welche Auswirkungen ergeben sich insbesondere im Hinblick auf das Widerspruchsrecht aus Art. 21 DSGVO für die Forschung, wenn besondere Kategorien personenbezogen Daten (z.B. Gesundheitsdaten) verarbeitet werden, wenn eine Verarbeitung auf Grundlage von Art. 6 Abs. 1e) oder f) erfolgt?

Es macht im Ergebnis keinen Unterschied im Hinblick auf die Behandlung des Widerspruchsrechts, auf welcher rechtlichen Grundlage die Verarbeitung der Daten erfolgt. (III Kap. 12.7) 
2. Kann sich die Rechtsgrundlage im Laufe der Verarbeitung verändern? Kann z.B. bei Widerruf einer Einwilligung ggf. noch eine andere Rechtsgrundlage anzuwenden sein, die eine weitere Verarbeitung erlaubt?

Es kann mehrere Rechtsgrundlagen geben, auf denen eine Datenverarbeitung beruht. Eine Veränderung der Rechtsgrundlagen kann sich dadurch ergeben, dass mit einer Datenverarbeitung zusätzliche andere Zwecke verfolgt werden. Die Berufung aufeine Rechtsgrundlage durch den Verantwortlichen hat für den Betroffenen die Funktion, die Rechtmäßigkeit einer Verarbeitung beurteilen und prüfen zu können. Der Wechsel der Berufung von einer Einwilligung zu einer gesetzlichen Rechtsgrundlage ist nur im Ausnahmefall unter Beachtung des Crundsatzes von Treu und Glauben möglich. Ein Wechsel ist unzulässig, wenn damit die Vertrauenserwartungen des Betroffenenverletzt werden. (III Kap. 7.3).

2.1 Welche Auswirkungen hat dies auf die Transparenzverpflichtungen (s. Art. $12 \mathrm{ff}$. DSGVO) und hier die notwendige Nennung der Rechtsgrundlage? Muss z.B. in einer Einwilligung explizit auf mögliche ergänzende Rechtsgrundlagen hingewiesen werden?

Soll esschon bei Einholung der Einwilligung möglich sein, die Berufung auf die Rechtsgrundlage zu wechseln, so muss hierauf und auf die Folgen für den Betroffenen hingewiesen werden. Ergibt sich im Nachhinein die Notwendigkeit eines zulässigen Wechsels, so besteht zum frühestmöglichen Zeitpunkt die Notwendigkeit einer Information. (III Kap. 7·3, Kap. 12.1)

\section{Verantwortlichkeit und Zusammenarbeit}

3. Für die Übermittlung von personenbezogenen sowie von personenbeziehbaren Daten bestimmen in der DSGVO die Regelungen zur gemeinsamen Verantwortlichkeit (Art. 26) sowie zur Auftragsverarbeitung (Art. 28) mögliche Rahmenbedingungen neben der Einzelverantwortlichkeit.

3.1 Wie sind die Regelungen zur gemeinsamen Verantwortlichkeit, zur Auftragsverarbeitung und zur Einzelverantwortlichkeit voneinander abzugrenzen?

Die gemeinsame Verantwortlichkeit lässt sich von der Auftragsverarbeitung dadurch abgrenzen, dass die Zwecke nicht allein durch einen Verantwortlichen festgelegt werden und keine verpflichtenden Weisungen ergehen können, sondern eine verbindliche Vereinbarung über die gemeinsamen Verarbeitungsschritte zwischen den verarbeitenden Stellen getroffen werden muss. Eine Einzelverantwortlichkeit besteht, wenn die Zwecke ausschließlich von einer Stelle festgelegt werden. Dies gilt auch bei Verarbeitungsketten, wenn die jeweilige Entscheidung über die Verarbeitung unabhängig ist von der vorangegangenen und folgenden Verarbeitung.

3.2 Welche Regelungsbedarfe sind in den jeweiligen vertraglichen Abreden besonders wichtig oder charakterisieren diese?

Die wesentlichen Inhalte eines Auftragsvertrags werden abschließend in Art. 28 DSGVO Abs. 3 aufgeführt. Mindestinhalte einer Vereinbarung über eine gemeinsame Verantwortlichkeit gemäßArt. 26 DSGVO sind Absprachen über die Behandlung von Betroffenenrechten, die Arbeitsteilung bei den Informationspflichten und die Festlegung der für Verarbeitungsverzeichnis relevanten Informationen gemäßArt. 30 Abs. 1 DSGVO.

3.3 Welche Vor- und Nachteile bieten die drei unterschiedlichen Varianten? Nach welchen Kriterien sollte der passende Regelungsansatz für einen praktischen Anwendungsfall bestimmt oder ausgewählt werden (z.B. in Form einer Checkliste darstellbar)? 
Ob eine Einzelverantwortlichkeit oder einer gemeinsame Verantwortung besteht, ist abhängig von der tatsächlichen Verarbeitung. Werden gemeinsame Zwecke verfolgt, so ist die Vereinbarung einer gemeinsamen Verantwortung naheliegend; wenn dabei die Verarbeitungsschritte voneinander abhängig sind und sich ergänzen, ist eine gemeinsame Verantwortung zwingend.

Vorteile der Auftragsverarbeitung: einseitige Festlegung der Zwecke und der Bestimmung der Rechtsgrundlagen, Übertragung der technischen Verarbeitung an einen Dienstleister, Weisungsmöglichkeit durch den Verantwortlichen, klare Trennung der datenschutzrechtlichen Pflichten.

Vorteile der gemeinsamen Verantwortlichkeit: differenziertere Arbeitsteilung, Eigenständigkeit der Beteiligten, Einbeziehung von mehr als zwei nicht hierarchisch eingebundenen Stellen. (III Kap. 5.2$5.7)$

3.4 Müssen ein Datentreuhänder und die juristische Person, die für ein Forschungsvorhaben verantwortlich ist (z.B. Universität), gemeinsam Verantwortliche nach der DSGVO sein oder kann wie bisher auch das Konstrukt der „Funktionsübertragung“ genutzt werden?

Es ist naheliegend, bei einer Einschaltung eines Treuhänders eine gemeinsame Verantwortlichkeit zu vereinbaren. Rechtlich möglich ist auch eine Auftragsverarbeitung, mit der aber die Vertraulichkeit der Treuhändertätigkeit beeinträchtigt würde. Eine Funktionsübertragung ist nicht zu empfehlen, da damit die Einbindung in einen gemeinsamen Zweck in Frage gestellt wäre. (II Kap. 5.9)

3.5 Wie ist im Verhältnis zu diesen Regelungsansätzen das früher mit dem Begriff der „Funktionsübertragung “ belegte Konstrukt generell einzuordnen?

Der Begriff der Funktionsübertragung ist entbehrlich. Er eignet sich aber weiterhin zur Abgrenzung von einer Auftragsverarbeitung. (III Kap. 5.8)

3.6 Führt eine gemeinsame Verantwortlichkeit nach Art. 26 automatisch - wenn keine andere Rechtsform für die Zusammenarbeit gewählt wird (z.B. eingetragener Verein) - zu einer Gesellschaft bürgerlichen Rechts nach deutschem Recht?

Die gemeinsame Verantwortlichkeit begründet bei Fehlen einer anderen Rechtsform nicht automatisch eine Gesellschaft bürgerlichen Rechts. Die Rechtsbeziehung zwischen den Verantwortlichen kann, soweit sie sich nicht aufdie Verantwortung für die Verarbeitung bezieht, frei gestaltet werden. (III Kap. 5.5)

\section{Auswirkung der Neufassung des $\S 203$ StGB auf Forschungsvorhaben}

4. In der medizinischen Forschung bestehen häufig Kooperationen zwischen niedergelassenen Ärzten und Forschungseinrichtungen. Wie muss vor diesem Hintergrund ein Anwendungsfall ausgestaltet sein, damit die Mitarbeiter einer Forschungseinrichtung „als sonstige mitwirkende Person“ des Arztes im Sinne des $\$ 203$ Abs. 3 Satz 2 StCB sind und Daten ohne Verletzung der ärztlichen Schweigepflicht übermittelt werden können?

Mitarbeitende einer Forschungseinrichtung sind dann Mitwirkende eines Arztes, wenn sie diesen in seiner Tätigkeit, die neben seiner Beratungs- und Behandlungstätigkeit auch seine Forschungstätigkeit einschließt, unterstützen und diese Unterstützung erforderlich ist. (II Kap. 6.6, Kap. 6.8)

4.1 Können die Mitarbeiter einer Forschungseinrichtung als „sonstige mitwirkende Person“ i.S.d. $\mathbb{2} 203$ Abs. 3 S. 2 StGB gelten, wenn sie einerseits den Arzt bei seiner Forschungstätigkeit unterstützen, andererseits ergänzend aber auch eigene Forschungsfragestellungen anhand der Daten bearbeiten? Könnte sich die Forschungs- 
einrichtung bei der Bearbeitung eigener Fragestellungen auf eine datenschutzrechtlich legitimierte Zweckänderung (z.B. $\mathbb{2} 27$ BDSG) berufen?

Mitarbeiter einer Forschungseinrichtung sind keine Mitwirkenden, soweit sie ausschließlich eigene Forschungsfragestellungen bearbeiten. (II Kap. 6.6, Kap. 6.8)

4.2 Ist eine Auftragsverarbeitung gemäß Art. 28 DSGVO als datenschutzrechtlicher Rahmen zwingend für eine Mitwirkung einer Forschungseinrichtung bei der forschenden Tätigkeit eines Arztes gemäß $\$ 203$ StCB?

Die Einordnung einer Datenverarbeitung als Auftragsverarbeitung, gemeinsame Verantwortlichkeit oder Datenübermittlung spielt für die Auslegung der beruflichen Geheimhaltungsregeln keine Rolle. (II Kap. 6.7)

\section{Datenschutzkonzept und Datenschutz-Folgenabschätzung}

5. Für medizinische Forschungsvorhaben, die mit sensiblen, personenbeziehbaren Gesundheitsdaten operieren, wird die Entwicklung und Abstimmung eines Datenschutzkonzepts empfohlen. Typischerweise enthalten solche Konzepte die Darstellung der Zwecke und Rechtsgrundlagen der Verarbeitung, die Regelung der Verantwortlichkeit, die Prozesse und Datenflüsse sowie insbesondere eine ausführliche Beschreibung der zum Schutz der Daten getroffenen technischen und organisatorischen Maßnahmen. Im Regelfall ist das Datenschutzkonzept damit auch Grundlage der in einem Verarbeitungstätigkeitenverzeichnis zu einem Projekt zu dokumentierenden Daten. Gesetzlich gefordert ist ein Datenschutzkonzept im Regelfall zwar nicht (für eine Ausnahme s. $\$ 75$ Abs. 1 SGB X), aber $\$ 22$ Abs. 2 BDSG bezieht sich beispielsweise im Wesentlichen auf die Inhalte eines Datenschutzkonzepts. Mit der DSGVO wurde der neue Begriff der Datenschutz-Folgenabschätzung (DSFA, Art. 35) eingeführt, der zwingend bei umfangreichen Verarbeitungen von besonderen Kategorien personenbezogener Daten (z.B. Gesundheitsdaten) einzuhalten ist (vgl. Art. 35 Abs. 3b) DSGVO).

5.1 In welchem Verhältnis stehen die bisherigen Inhalte eines solchen Datenschutzkonzepts und die genannten gesetzlichen Regelungsansätze zur neu geregelten DSFA?

Die Pflicht, ein Datenschutzkonzept für ein Forschungsvorhaben vorzulegen, besteht nicht generell, sondern nur gemäßeinigen speziellen Gesetzen. Eine entsprechende Pflicht kann generell allenfalls aus den Dokumentationspflichten nach Art. 5 Abs. 2 DSGVO abgeleitet werden. Eine präzise Festlegung der notwendigen Inhalte ergibt sich aus den Gesetzen nicht. Als den Datenschutz umfassendes Dokument sollte es das Verarbeitungsverzeichnis, die Darstellung der technisch-organisatorischen Maßnahmen, die Datenschutz-Folgenabschätzung, die Einschränkung der Betroffenenrechte und die kompensierenden Garantien sowie, soweit es hierauf ankommt, die nötigen Interessenabwägungen enthalten. (II Kap. 11.3, Kap. 11.4)

5.2 Gibt es aus rechtlicher oder ggf. auch praktischer Sicht Hinweise darauf, dass man notwendiger Weise zwei getrennte Dokumente braucht oder die beiden Texte in einem Dokument zusammengefasst werden sollten?

Die Datenschutz-Folgenabschätzung kann im Rahmen eines umfassenderen Datenschutzkonzeptes vorgenommen werden. Möglich sind auch separate Dokumente, wobei es dann sinnvoll ist, dass aufeinander Bezuggenommen wird. (III Kap. 11.4) 


\section{Betroffenenrechte}

6. Wie ist der Begriff der „durch den Betroffenen bereitgestellten“ Daten in Art. 20 DSGVO (Datenportabilität) im Kontext der wissenschaftlichen Forschung zu verstehen?

Bereitgestellte Daten des Betroffenen sind auch solche, die durch das Verhalten des Betroffenen generiert werden, nicht jedoch solche, die hieraus abgeleitet sind. (III Kap. 12.6)

6.1 Fallen „Messdaten“ (wie z.B. MRT-Bilder, EEG-Aufnahmen, Röntgenbilder), vom Betroffenen selbst abgegebene Biomaterialproben (z.B. Haarlocke, Blut) und durch Analysen daraus ermittelte genetische Daten unter den Tatbestand der „Bereitstellung durch den Betroffenen“?

Medizinische Messdaten und Bilder gehören ebenso wie Wearable-Daten zu den bereitgestellten Daten. Demgegenüber fallen Biomaterialproben nicht darunter, da es sich um Datenträger handelt und nicht um automatisiert verarbeitete Daten. Auch nicht darunter fallen die Ergebnisse von (genetischen) Analysen dieser Proben. (III Kap. 12.6)

6.2 Es wird teilweise vertreten, dass die Datenportabilität für öffentliche Stellen (z.B. staatliche Hochschulen) im Forschungskontext ausgeschlossen sein soll (vgl. Artikel Prof. Roßnagel, ZD 4/2019, S. 163). Welche Argumente sprechen für und welche gegen diese Auslegung, insbesondere in den Fällen, in denen öffentliche Stellen mit einer Einwilligung der Betroffenen Daten verarbeiten?

An privilegierten Forschungsvorhaben, nicht nur solchen von Hochschulen, besteht ein öffentliches Interesse, weshalb hieraufdie Regelung zur Datenübertragbarkeit nicht anwendbar ist. Soweit diese Voraussetzungen nicht gegeben sind, etwa bei kommerzieller Forschung oder bei einer Datenverarbeitung durch Krankenhäuser oder Arztpraxen, besteht grundsätzlich ein Anspruch auf Datenportabilität nach Art. 20 DSGV, soweit die Daten auf Crundlage einer Einwilligung oder eines Vertrags bereitgestellt sind. (III Kap. 12.6)

7. Wie umfassend ist das Auskunftsrecht nach Art. 15 DSGVO zu verstehen?

7.1 Müssen bei einem Auskunftsersuchen alle Einzeldaten eines Betroffenen herausgegeben werden?

7.2 Schließt dies auch in einem Forschungsprojekt erst später gewonnene personenbezogene und personenbeziehbare Analyseergebnisse mit ein?

Der Auskunftsanspruch ist allumfassend in Bezug auf Daten mit Personenbezug und schließt, über die Ausgangsdaten hinausgehend, auch die individualisierbaren Forschungsergebnisse mit ein. (III Kap. 12.3)

7.3 In welchen Fällen kann das Recht auf Auskunft in der Forschung gemäß \$27 Abs. 2 BDSG in Verbindung mit Art. 89 Abs. 2 DSGVO eingeschränkt werden? Wann sind die Voraussetzungen des Tatbestandes „,voraussichtlich die Verwirklichung der Forschungszwecke unmöglich machen oder ernsthaft beeinträchtigen“ erfüllt? Nennen Sie mögliche Beispiele.

Unmöglichkeit einer Auskunftserteilung ist gegeben, wenn eine Zuordnung zur anspruchstellenden Person, auch mit ihrer Unterstützung, nicht mehr möglich ist. Eine Auskunftserteilung macht ein Forschungsprojekt unmöglich, wenn im Fall einer Auskunftserteilung die legitime Zielsetzung des Projektes vereitelt würde. Ein Auskunftsverlangen beeinträchtigt ernsthaft die Verwirklichung der Forschungszwecke, wenn der dafür nötige Aufwand die zumutbare Kapazität des oder der Forschenden übersteigt, so dass ein Projekt nicht abgeschlossen werden kann. Für den Aufwand ist relevant, wie viele Betroffene es gibt, welchen Aufwand die jeweiligen Zuordnungen und die Glaubhaftma- 
chungen der Identität verursachen, wie umfangreich die Datensätze sind und welche Kommunikationsform mit den Betroffenen möglich ist. (II Kap. 12.3)

7.4 Wie verhält sich das Recht auf Nichtwissen im Gegensatz zu den Auskunftsrechten der DSGVO? Müssen beispielsweise Patienten über für sie nicht vorhersehbare genetische Risikomarker für Demenz aufgrund eines Auskunftsersuchens nach Art. 15 DSGVO informiert werden? Oder dürfen solche sensiblen Informationen trotz eines Auskunftsersuchens zurückgehalten werden (s.a. Frage 7.3)? Welche Rolle spielt für die Umsetzung der Auskunftsrechte bei genetischen Daten in Forschungsprojekten das Gendiagnostikgesetz?

Die verfassungsrechtlich begründeten Rechte auf Nichtwissen bzw. auf Teilwissen, für die es keine ausdrückliche Erwähnung in der DSGVO gibt, sind bei Auskunftserteilungen zu berücksichtigen. Hierüber hat letztlich der Betroffene - eventuell nach einer eingehenden Beratung - selbst zu entscheiden. Die Regelungen des GenDG sind direkt oder entsprechend anzuwenden. Um das Recht auf Nichtwissen Dritter (z.B. biologischer Verwandter) zu wahren, ist der Betroffene aufentsprechende Implikationen bei der Auskunftserteilung hinzuweisen. (III Kap. 12.3)

8. In welcher Form kann den Transparenzanforderungen nach Art. 13 (und Art. 14) entsprochen werden, wenn die Verarbeitung nicht auf Basis einer informierten Einwilligungserklärung erfolgt? Wie müssen beispielsweise Betroffene dementsprechend über die Nutzung ihrer Daten zu Forschungszwecken informiert werden, wenn diese auf Basis der Forschungsregelungen in einem Landeskrankenhausgesetz erfolgt? Wie muss über ggf. später erfolgende Änderungen der gesetzlich geregelten Rechtsgrundlagen und sich daraus ergebende weitere Verarbeitungen informiert werden?

Die Informationspflichten werden in den Art. 12-14 DSGVO detailliert beschrieben. Sind Sekundärnutzungen, etwa auf Crundlage eines Landeskrankenhausgesetzes, bei der Erhebung bekannt, muss zu diesem Zeitpunkt informiert werden; ansonsten ist eine frühestmögliche Information, in jedem Fall vor der Verarbeitung, nötig. Die Voraussetzungen für einen Verzicht auf eine nachträgliche Information sind in Art. 14 Abs. 5 DSGVO beschrieben. (III Kap. 12.1)

9. Wie ist den Auskunftsrechten nach Art. $14 \mathrm{zu}$ entsprechen, wenn die verantwortliche Stelle oder ein Auftragsverarbeiter die Daten im Forschungskontext beispielsweise lediglich pseudonym verarbeitet und keinen Zugriff auf eine Zuordnungsliste hat?

Ist es einem Verantwortlichen z.B. wegen einer ausschließlich pseudonymen Verarbeitung, nicht möglich, einem Betroffenenanspruch nach den Art. 12ff. DSGVO zu entsprechen, dann ist er hierzu auch nicht verpflichtet. Dies gilt auch für eine Information nach Art. 14 DSGVO und die Auskunft nach Art. 15 DSGVO. Unmöglichkeit ist aber nicht gegeben, wenn der Betroffene die zur Identifikation erforderlichen Informationen nachliefert (Art. 11 Abs. 2 S. 2 DSVO) oder wenn für den Verantwortlichen eine Zuordnung mit Hilfe eines Projektbeteiligten möglich ist. (III Kap. 12.3)

\section{Sonderregelungen für die Forschung}

10. Welche Konsequenzen ergeben sich aus dem Recht auf Löschung nach Art. 17 Abs. 3 lit. d DSGVO für die Aufbewahrung von Daten, die bereits im Rahmen einer wissenschaftlichen Publikation nach bisheriger Rechtslage veröffentlicht wurden?

Gemäßden Regelungen in deutschen Forschungsklauseln dürfen personenbezogene Daten im Rahmen der Forschung nur veröffentlicht werden, wenn der Betroffene eingewilligt hat oder dies für die 
Darstellung der Forschungsergebnisse über Ereignisse der Zeitgeschichte unerlässlich ist. Möglich ist, dass die öffentliche Erreichbarkeit von Daten aus Gründen des Persönlichkeitsschutzes eingeschränkt werden muss. Sind die Voraussetzungen für eine zulässige Veröffentlichung gegeben, so dürfen veröffentlichte Forschungsdaten weitergenutzt werden. Fehlt es hieran, so ist auch die Weiterverwendung regelmäßig unzulässig. (III Kap. 12.8)

10.1 Könnte diese Norm beispielsweise die weitere Aufbewahrung von Daten nach Widerruf/Löschungsverlangen rechtfertigen, wenn aufgrund der Daten bereits publiziert wurde und die gute wissenschaftliche Praxis zwecks Nachvollziehbarkeit von veröffentlichten Arbeiten eine Aufbewahrung von 10 Jahren empfiehlt?

Die Notwendigkeit, Forschungsergebnisse überprüfen zu können, kann die weitere Rechtfertigung von Daten legitimieren, selbst wenn Betroffene die Löschung verlangen oder der Verarbeitung widersprechen. Voraussetzung ist, dass die weitere Speicherung hierfür erforderlich ist. Rechtsgrundlage für die weitere Speicherung ist die ursprüngliche Rechtsgrundlage. Erforderlich ist die Speicherung in vielen dieser Fälle nur in pseudonymer Form. (Kap. 7.3, Kap. 12.8, Kap. 12.9)

11. Welche Rechte gewährt Art. 17 Abs. 3 lit. d DS-GVO dem Verantwortlichen generell? Inwieweit ist Art. 11 DSGVO im Forschungskontext anwendbar, beispielsweise bei pseudonymer Verarbeitung der Daten? Mit welchen konkreten Folgen/Erleichterungen (s. auch Frage 9)?

Bei der Datenverarbeitung für Forschungszwecke sind die Verantwortlichen nach Art. 89 Abs. 1 DSGVO zu einer frühestmöglichen Anonymisierung oder Pseudonymisierung verpflichtet. Können sie deshalb die Daten der Betroffenen nicht mehr zuordnen, so muss Betroffenenansprüchen nicht mehr entsprochen werden, wenn nicht der Betroffene zusätzliche Informationen bereitstellt, mit denen die Identifikation möglich ist. Nach Art. 17 Abs. 3 lit. d DSGVO kann auf eine Löschung, Anonymisierung oder Pseudonymisierung nur verzichtet werden, wenn damit die Verwirklichung der Ziele des Forschungsprojektes unmöglich oder ernsthaft beeinträchtigt würden. (III Kap. 12.3, Kap. 12.8)

12. In Forschungsprojekten, in denen die erhobenen Daten relativ langfristig und für offene Forschungsfragen zur Verfügung gestellt werden, besteht im Sinne einer informationellen Gewaltenteilung die Anforderung, die Identitätsdaten und das Pseudonymmanagement unabhängig von der Verwaltung der eigentlichen Forschungsdaten im Sinne einer unabhängigen Treuhandstelle zu organisieren.

12.1 Besteht diese Anforderung der „informationellen Gewaltenteilung“ auch nach der DSGVO und woraus leitet sie sich ab?

Das vom deutschen Bundesverfassungsgericht aus dem Grundgesetz abgeleitete Instrument der „informationellen Gewaltenteilung“ ist auch unter der Anwendung europäischen Rechts weiterhin relevant, da der europäische Grundrechteschutz dem nationalen entspricht. Abweichungen durch die europäische Rechtsprechung sind nicht erkennbar. (III Kap. 10.4)

12.2 Manchmal wird in solchen Fällen, insbesondere wenn die Daten nur aus einer Einrichtung kommen, keine externe Stelle genutzt, sondern die Treuhandfunktion dem behördlichen oder betrieblichen Datenschutzbeauftragten der verantwortlichen Stelle übertragen. Ist beim Vorliegen einer solcherart angelegten Doppelfunktion als Treuhandstelle und Datenschutzbeauftragter eine eigene Aufsicht für den Datenschutzbeauftragten notwendig bzw. nach welchen rechtlichen Kriterien und Gesetzen kann diese Frage beantwortet werden? 
Bei der gleichzeitigen Wahrnehmung der Funktion eines Datenschutzbeauftragten und eines Datentreuhänders kann es zu Interessenkonflikten kommen, weshalb eine solche Verbindung nicht zu empfehlen ist (II Kap. 10.4)

13. Auf welcher Rechtsgrundlage dürfen bereits unter früherem Recht erhobene und verarbeitete Biomaterialproben (sog. „Altproben“), die nach altem BDSG als anonym betrachtet werden konnten, nach der DSGVO und ggf. dem neuen BDSG verarbeitet werden? Was ergibt sich, wenn zu diesen Altproben keine direkt identifizierenden Daten einzelner Personen oder Patienten mehr gespeichert bzw. zugreifbar sind?

Altproben, die nach früherem Recht als anonym eingestuft wurden, unterliegen dem aktuellen Datenschutzrecht und der DSGVO, wenn sie zum Zeitpunkt der Verarbeitung dadurch personenbeziehbar sind, dass sie durch potenziell verfügbares Zusatzwissen einer Person zugeordnet werden können. (II Kap. 10.1)

14. Es besteht zurzeit große Unsicherheit darüber, welche Rechtsgrundlagen anzuwenden sind, wenn Daten und ggf. Biomaterialproben aus einem Nicht-EU-Land (z.B. USA), in dem sie als anonym klassifiziert werden, an einen Forschungspartner in der EU geschickt werden und europäische Forschungseinrichtungen indes - auf Grundlage der DSGVO - von einem Personenbezug ausgehen müssten. Kann die rechtliche Qualifizierung an der Quelle als „anonym“ unter der DSGVO weiter gelten? Wie sind Fälle zu beurteilen, in denen eine Zuordnung zu einer Person oder einem Patienten nur im Nicht-EU-Ausland möglich ist?

Durch die Vorgaben der DSGVO und deren Präzisierung durch den EuGH ist in abstrakter Weise klargestellt, wann eine Anonymisierung von Daten anzunehmen ist. Entscheidend ist der Aufwand, die Daten einer konkreten natürlichen Person zuzuordnen, und damit die Wahrscheinlichkeit, dass dies möglich ist. Eine anderweitige Bewertung in einem Nicht-EU-Land spielt für die Verarbeitung durch Stellen in der EU keine Rolle. Ist das für eine Identifizierung nötige Zusatzwissen ausschließlich im Drittausland verfügbar und ist es völlig unwahrscheinlich, dass dieses Zusatzwissen verfügbar gemacht werden kann, dann kann Anonymität angenommen werden. (II Kap. 10.1, Kap. 13.6)

\section{Evaluation der Forschungsvorschriften aus der EU-DSGVO}

15. Bewerten Sie bitte den aktuellen Regelungsrahmen der DSGVO mit Blick auf die konkrete Anwendbarkeit in der medizinischen Forschung. Nutzen Sie hierfür die Analysen und Antworten zu den vorangehenden Fragen. Gehen Sie bei engem Bezug zur DSGVO, und soweit aus Ihrer Sicht die Notwendigkeit besteht, auch auf relevante Regelungen auf Bundes- und Landesebene ein.

15.1 Welche positiven Ansätze sehen Sie?

Die DSGVO bietet einen validen europarechtlichen Rahmen für einen Ausgleich zwischen dem Datenschutz und der Forschungsfreiheit, indem sie einerseits die Datenverarbeitung für Forschungszwecke privilegiert, andererseits geeignete Garantien für die Betroffenen notwendig macht. (II Kap. 14.1)

15.2 Welche Regelungen sind aus Ihrer Sicht problematisch?

Dadurch, dasses die DSGVO über Öffnungsklauseln weitgehend den Mitgliedstaaten überlässt, die materiellen Regelung zur Datenverarbeitung, die organisatorischen und prozessualen Rahmenbedingungen sowie die Beschränkung der Betroffenenrechte zu regeln, besteht bisher kein einheitliches Datenschutzrecht für Forschungszwecke in der Europäischen Union. Die Gesetzgeber in Deutschland haben die Öffnungsklauseln bisher nicht dafür genutzt, eine inhaltliche Anpassung an die europäischen Vorgaben und eine nationale Harmonisierung vorzunehmen. Die Regeln genügen zudem nicht 
den Anforderungen an eine moderne Forschungstätigkeit. Die bestehende Rechtsunsicherheit ist hinderlich sowohl für eine wirksame Forschungstätigkeit wie für den Datenschutz. (II Kap. 14.1) 15.3 Welche Auslegungsprobleme oder Unklarheiten in der Anwendung sehen Sie?

Es ist unklar, welche Forschungsprojekte als privilegiert behandelt werden können. Die Unbestimmtheit der Regeln geben den Forschenden keine klaren Vorgaben. Es fehlt sowohl an Standards wie auch an einer einheitlichen Infrastruktur für Transparenz und wissenschaftliche Kooperation. (III Kap. 14.2)

15.4 Welche Empfehlungen würden Sie dem europäischen Gesetzgeber sowie ggf. auch den nationalen Gesetzgebern auf Bundes- und Landesebene für die Weiterentwicklung des Rechtsrahmens mit auf den Weg geben?

Aufder Grundlage der DSGVO kann auf europäischer Ebene ein einheitliches Datenschutzrecht für die Forschung oder zumindest für die medizinische Forschung geschaffen werden, zumal es über die wesentlichen Regelungsinhalte einen weitgehenden Konsens geben dürfte. Solange eskeine europarechtlichen Regelungen gibt, sind die deutschen Gesetzgeber gefordert, auf nationaler Ebene einheitliche Regelungen zu schaffen. Dies wäre - aber erst nach einer Änderung der Gesetzgebungszuständigkeit - durch ein Bundesgesetz möglich. Ohne Grundgesetzänderungbietet sich der Abschluss eines Bund-Länder-Staatsvertrags an. Bis dahin und zur Vorbereitung einer Gesetzgebung wird die Ausarbeitung von Verhaltensregeln empfohlen (III Kap. 14.2) 